\title{
Dynallax: Solid State Dynamic Parallax Barrier Autostereoscopic VR Display
}

\author{
Tom Peterka ${ }^{1}$, Robert L. Kooima ${ }^{1}$, Javier I. Girado ${ }^{1}$, Jinghua $\mathrm{Ge}^{1}$, Daniel J. Sandin ${ }^{1,2}$, Andrew Johnson ${ }^{1}$, \\ Jason Leigh ${ }^{1}$, Jurgen Schulze ${ }^{2}$, Thomas A. DeFanti ${ }^{1,2}$ \\ ${ }^{1}$ Electronic Visualization Laboratory \\ University of Illinois at Chicago \\ ${ }^{2}$ California Institute for Telecommunications and Information Technology \\ University of California at San Diego
}

\begin{abstract}
A novel barrier strip autostereoscopic (AS) display is demonstrated using a solid-state dynamic parallax barrier. A dynamic barrier mitigates restrictions inherent in static barrier systems such as fixed view distance range, slow response to head movements, and fixed stereo operating mode. By dynamically varying barrier parameters in real time, viewers may move closer to the display and move faster laterally than with a static barrier system. Furthermore, users can switch between 3D and 2D modes by disabling the barrier. Dynallax is head-tracked, directing view channels to positions in space reported by a tracking system in real time. Such head-tracked parallax barrier systems have traditionally supported only a single viewer, but by varying the barrier period to eliminate conflicts between viewers, Dynallax presents four independent eye channels when two viewers are present. Each viewer receives an independent pair of left and right eye perspective views based on their position in 3D space. The display device is constructed using a dual-stacked LCD monitor where a dynamic barrier is rendered on the front display and the rear display produces a modulated VR scene composed of two or four channels. A small-scale head-tracked prototype VR system is demonstrated. Performance data are analyzed while advantages, disadvantages, ongoing and future work are identified.
\end{abstract}

CR categories: I.3.7 [Computer Graphics]: Three-Dimensional Graphics and Realism --- virtual reality

Keywords: autostereoscopic display, 3D display, virtual reality, Dynallax, Varrier, parallax barrier

\section{INTRODUCTION}

Lenticular screens and parallax barrier strip displays are dominant and popular autostereoscopic (AS) technologies [7] [13]. Published literature on lenticular and barrier strip AS abounds, and any internet search quickly reveals the myriad commercial products that are available. In 2004, the Electronic Visualization Laboratory (EVL) at the University of Illinois at Chicago (UIC) in conjunction with IEEE VR'04 presented its barrier strip AS display system named Varrier [11].

address correspondence to: tpeterka@evl.uic.edu
In this paper, Varrier serves as the specific testbed against which Dynallax is compared, but the results are extendable to other head-tracked static barrier systems and to that class of AS technology in general. Most people who experience Varrier are satisfied with the overall experience, but can also quickly point out these shortcomings: limited spatial resolution, restrictions on head movement speed, and the fact that only one user can view AS at a time. Inherent to a static barrier, these problems have not been overcome within the confines of that methodology.

A dynamic barrier addresses some of those shortcomings and has other advantages as well. Specifically, the benefits of Dynallax are: expanded view distance working range, reduced sensitivity to system latency during head movement, eliminated physical barrier registration, ability to disable the barrier and convert the display to $2 \mathrm{D}$, and the affordance of two independently tracked viewers, each with their own AS perspective of the virtual world.

Dynallax imposes a few complications of its own. For example, the physical nature of the dynamic barrier produces lower contrast and brightness, while the color sub-structure of the LCD used for the front barrier physically limits algorithmic resolution gains. These limitations are discussed near the end of the paper, and research is ongoing to address these physical constraints imposed by the underlying LCD technology.

\section{BACKGROUND}

The function of a parallax barrier is to occlude certain regions of an image from each of the two eyes, while permitting other regions to be visible, as in Fig. 1.

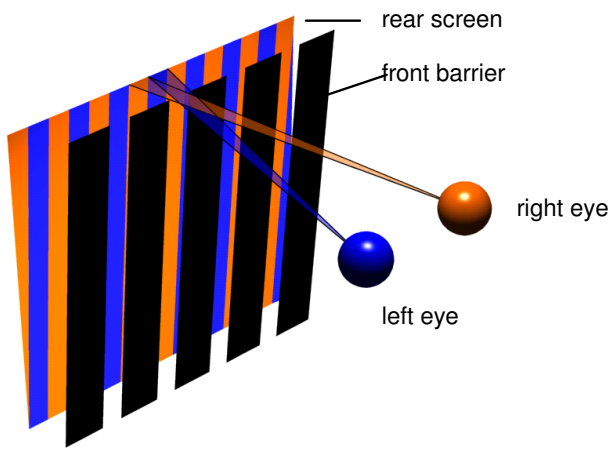

Figure 1: A parallax barrier is a series of transparent / opaque strips that selectively transmit / occlude corresponding image strips to / from each eye. 
By simultaneously rendering strips of a left eye image into the regions visible by the left eye and likewise for the right eye, a complete perspective view is directed into each eye. Then, by fusing two stereo images together into a 3D representation, an AS experience results without the need for 3D glasses.

When the barrier strip concept is coupled with real-time viewupdate, head-tracking, first-person perspective, and interactive application control, an AS VR system results. Figure 2 illustrates two implementations of the Varrier AS VR system in large and small form factors.

The parallax barrier is a high-resolution printed film that is affixed to a glass substrate and appended to the front of an LCD monitor. [12] Another popular variation is the lenticular screen; both function equivalently. [5] The printed pattern on the barrier is a fine-pitch sequence of opaque and transparent strips; the period of this repeating pattern is on the order of .5 to $1 \mathrm{~mm}$.

The period of this barrier is determined a priori by the system designer and determines key outputs in system response that cannot be varied once built. Such output parameters as view distance operating range (minimum, maximum, optimum), visual acuity, and the fact that the system is capable of only supporting one user at a time are three such results of barrier period choice. The consequences of these design-time decisions are magnified by the long turn-around time to correct or modify the barrier. Moreover, with respect to supporting two tracked viewers, there is no single optimal barrier period that can be pre-selected; in Sections 3 and 4 it will be shown that the barrier period must vary in real time to prevent inter-channel conflicts.

In addition to fixed working range and strict single user mode for tracked two-view systems, static barrier AS systems have some other disadvantages. One is that the barrier cannot be disabled, affording a convertible 3D / 2D system such as [14]. Another is horizontal resolution loss, since one barrier period consists of a duty cycle of approximately $3 / 4$ black to $1 / 4$ clear. Hence, each eye channel contains only $1 / 4$ of the horizontal screen resolution. The spatial inefficiency of parallax barrier AS is a direct result of the Nyquist Sampling Theorem, which dictates that eye channels are separated by equivalent amounts of unused screen space, termed "guard bands."

Finally, head-tracked static barrier AS is further limited by the fact that performance criteria such as frame rate and latency are more critical in fixed barrier AS than in other stereo techniques. Unlike passive and active stereo VR, moving the head faster than the system response time results not only in scene lag but also in visible artifacts because incorrect data is steered to the eyes. Since channels are continuously steered to the viewer's eyes in headtracked AS, one may readily out-run the system and move the head faster than channels can be updated. Defects such as image flicker, black banding, and ghosting are visible in a head-tracked AS VR system during head movements, and disappear when the viewer stops moving. The goal of Dynallax is to improve these system limitations through the use of a dynamic barrier.
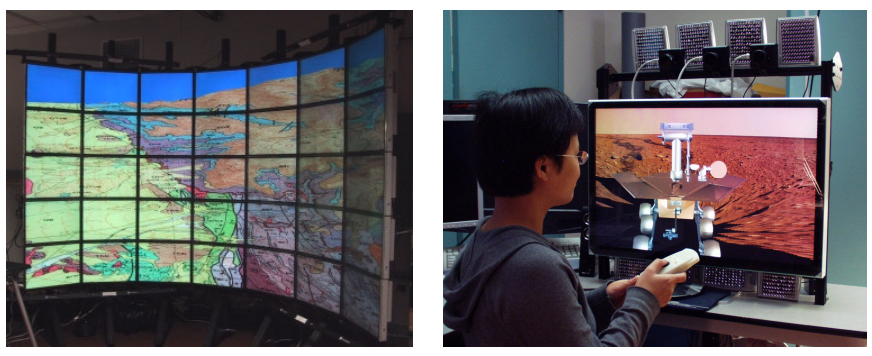

Figure 2: The Varrier AS VR system is shown in large tiled (left) and smaller desktop (right) versions.
Dynamic parallax barriers have appeared in AS literature in various contexts. For example, Moseley et al. [16] proposed an electro-mechanical combination of barriers to produce dynamic results in response to head-tracked viewer positions. Other systems include solid-state dynamic barriers similar to Dynallax, but the purpose of the barrier is to solve other problems than those addressed here. The Cambridge Display [17] time-multiplexes a number of channels to pre-determined untracked positions in space using a ferroelectric liquid crystal shutter. The NYU Display [18] utilizes a rapidly oscillating barrier rendered on a picell ferroelectric liquid crystal shutter. By combining spatial multiplexing inherent in a parallax barrier with the time multiplexing of 3 barrier phases, this system increases spatial resolution and simultaneously conceals the coarse scale of the barrier strips.

\section{SYSTEM DESCRIPTION}

\subsection{Dynamic barrier construction}

A dual-stacked LCD display can be constructed by placing a second liquid crystal (LC) layer in front of an existing LCD display, such that both layers are illuminated by a single backlight, and the front layer angle of polarization is orthogonal to the rear. The final output intensity is the product of the two individual layer intensities:

$$
\mathrm{I}_{\text {final }}=\mathrm{I}_{\text {rear }} * \mathrm{I}_{\text {front }}
$$

A complete dual-stacked display is available from [9], and this is the display used for the Dynallax prototype.

\subsection{System Structure}

Dynallax is structured as a small cluster of three processes: a master controller, the front screen rendering slave, and the rear screen rendering slave. Inter-process communication is accomplished with MPICH 2.0 [6]. Currently each process resides on a separate Linux machine with dual Intel Xeon CPUs, an NVIDIA Quadro FX3000 GPU, and gigabit Ethernet.

The front and rear screens of the dual-stacked display cannot be driven from the same graphics card because most Dynallax modes require asynchronous update of the two screens. The front screen is lightly loaded compared to the rear, since the front needs to only render the barrier while the rear screen renders the modulated scene.

Head tracking is accomplished using an Intersense 900 [2] tracking system with two sensors, for two viewers. Each viewer wears one sensor on a headband on the forehead. Later implementations will utilize tether-less camera tracking, as currently included in the Varrier display [1]. At this point in the research however, tethered sensors are sufficient for testing and as a proof of concept, as in Figure 3 which depicts one user wearing a tracking sensor on a headband, interactively navigating through a VR scene.

An independent first person perspective is channeled to each of up to two viewers. Real time interactivity is afforded with either a tracked or untracked wand containing a joystick and multiple buttons. In the case of two viewers, the wand is shared or controlled by only one viewer at a time. 


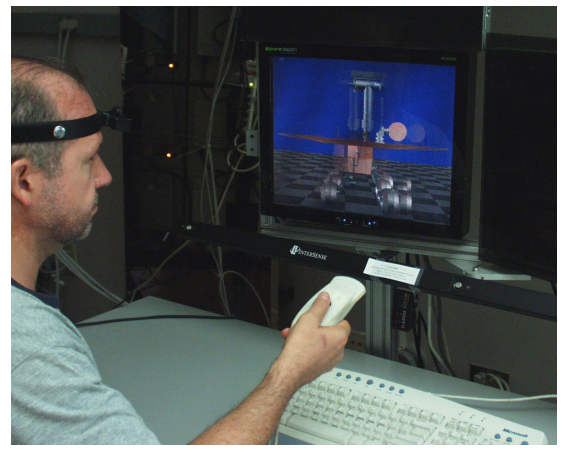

Figure 3: A user interacts with Dynallax, wearing a tracking sensor on a headband. Eventually, tracking will be camera-based and tetherless, as in other systems at EVL.

\subsection{Image Computation Algorithm}

The same computational model is used to render the front barrier as to modulate the rear screen channels, which is the process of taking two or four perspective views and interleaving alternating strips of each into a single image. In order to modulate the rear screen scene, and to render the front visible barrier, a constantly variable floating point step function is constructed of the desired period and duty cycle. This function determines whether a subpixel fragment is masked as white or black. In the case of the rear screen, this mask is multiplied by the rear scene content, while in the front screen, this mask is the front barrier intensity.

The method is conceptually equivalent to the Varrier Combiner algorithm [3], [4]. The main differences between the algorithms is that Dynallax always uses a constant quality level of 1.0 [4] and twice as many eye channels are supported, permitting two viewers to modulate a total of four eye channels.

There is a fundamental distinction between this approach and other sub-pixel barriers such as [13] and [15]. Instead of utilizing a discrete image-based barrier that is sub-pixel registered with the underlying sub-structure of the display device, the barrier computational model in Dynallax is continuous and floating-point based. This is by design, and there are several advantages to this approach. For example, not only is the barrier period continuously adjustable, but so is the line tilt angle, so barrier parameters are infinitely variable to dynamically optimize viewing conditions. Moreover, when the barrier is scaled by perspective projection from the front to the rear screen, quantization errors do not result from the multiple discretization that would occur if the barrier originated as an image-based (discrete) model. Rather, a scaled continuous barrier model is used to modulate the rear screen, and is discretized only once at the end of the process when converted to visible pixels by the graphics card.

\subsection{Controller algorithm}

A real-time controller sets the barrier period, duty cycle, and barrier shift at each frame update. The controller contains three modules, as shown in Figure 4. Each of these modules is described in this section.

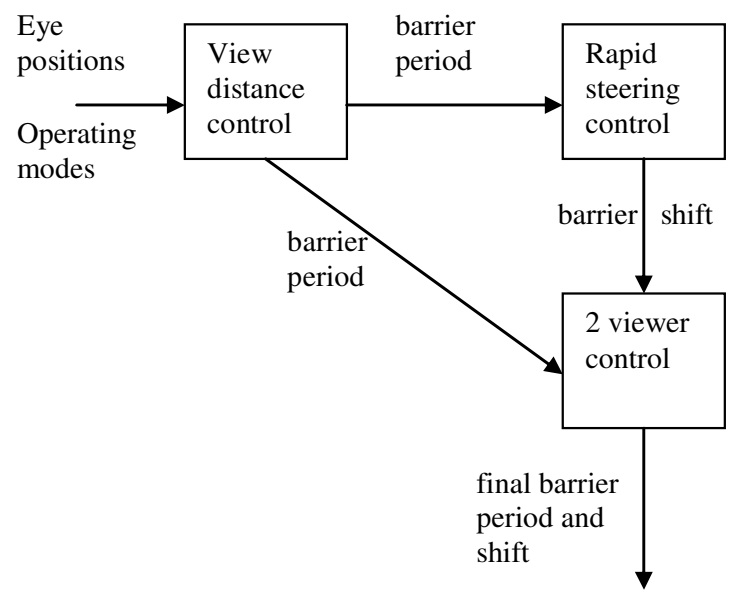

Figure 4: Controller block diagram illustrates three main functions: view distance, rapid steering, and 2-viewer control.

\section{View distance control}

At the optimal viewing distance from the screen in a static barrier system, eye channel data and guard bands are spaced equidistantly within the rear screen modulated image. This optimal view distance is a function of the barrier period. In Dynallax, the view distance control module sets the barrier period such that this condition is always satisfied according to equation 2:

$$
\mathrm{p} / \mathrm{t}=(2 \mathrm{e}-\mathrm{p}) /(\mathrm{d}+\mathrm{t})
$$

where:

$\mathrm{p}=$ barrier period

$\mathrm{t}=$ optical thickness between front and rear screens

$\mathrm{e}=$ interocular distance

$\mathrm{d}=$ normal distance from eyes to front screen plane

By constantly maintaining an optimal barrier period for the current viewer distance from the screen, the system not only maintains the best possible image quality but also permits the viewer to be closer to the screen than would otherwise be permitted by the minim view distance for a fixed system [12].

\section{$\underline{\text { Rapid steering control }}$}

In traditional fixed barrier AS, it is easy to produce head movements fast enough to outrun the system response, resulting in momentary incorrect steering of channels to the eyes. When this occurs, the viewer sees black banding because the eyes pass into the guard band regions before the scene can be updated. This is a common occurrence in Varrier, for example. The purpose of rapid steering is to weaken the dependence of stereo quality on system latency, ideally affecting only perspective correctness as in the case of passive stereo.

In Dynallax, the front screen has a constant computational complexity and is relatively lightly loaded, maintaining a $50 \mathrm{~Hz}$ frame rate. This is compared to the rear screen whose complexity depends on the VR scene complexity and drops to $15 \mathrm{~Hz}$ or slower for complex scenes on the order of $100 \mathrm{~K}$ vertices. The reasons for this disparity between front and rear loading are not technical limitations of Dynallax; rather, they are a reflection of VR usage in general. Scene complexity grows as data set sizes 
increase, and frame rate is often sacrificed for higher image quality. These performance pressures only impact the rear screen in Dynallax, however.

Furthermore, it is exactly this disproportionate load that makes a rapid steering mode possible, where rapid head movements are accommodated by shifting the rendered front barrier rather than waiting to re-render the rear image. The rear screen continues its computation, but the front and rear screens are de-coupled allowing both screens to proceed asynchronously at their fastest rates possible. The rapid steering control module monitors this behavior and sets the barrier shift according to equation 3 .

$$
\mathrm{s}=\mathrm{e}_{\mathrm{x}} * \mathrm{t} /(\mathrm{t}+\mathrm{d})
$$

where:

$\mathrm{s}=$ barrier shift amount

$\mathrm{e}_{\mathrm{x}}=$ horizontal head movement distance

$\mathrm{t}=$ optical thickness between front and rear screens

$\mathrm{d}=$ normal distance from eyes to front screen plane

\section{$\underline{2 \text { viewer barrier control }}$}

Thus far the explanation of the controller algorithm has focused on features primarily used when only one viewer is present, view distance control and rapid steering. Nevertheless, a main feature of the system is support for two tracked viewers, each receiving an independent pair of perspective eye channels. The ensuing discussion now turns to how the controller sets the barrier period in two-viewer mode.

When two viewers are present, the barrier period must increase for two reasons. First, the period must expand by at least a factor of two to produce room for the additional channels to be multiplexed into the final image. Beyond this mandatory expansion, the period may need to grow further to avoid a conflict between each actual eye and the virtual repeating lobes from the other eyes.

To understand this concept, one must realize that a parallax barrier display does not only direct optical channels to the two eye positions; rather those channels are repeated at multiple recurring locations across space as shown in Figure 5. Here, the term "virtual lobe" is used to distinguish the primary eye channel location from all of its repetitions in space.

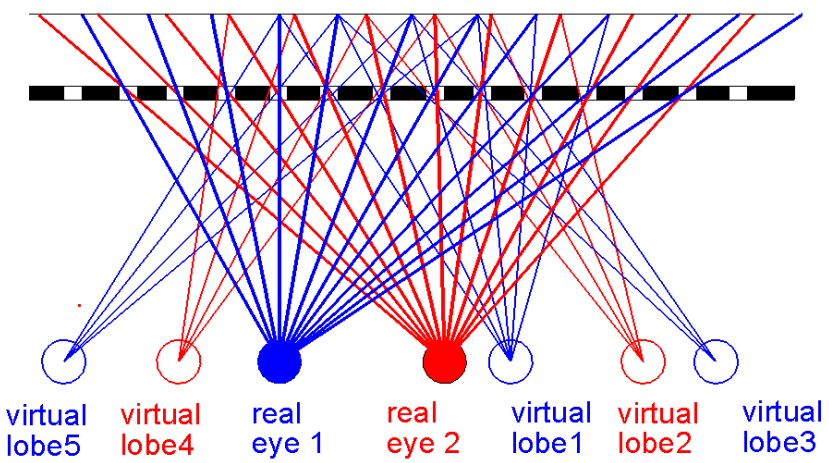

Figure 5: Repetition of channel lobes in space occurs at regularly spaced intervals. Although a limited number of lobes are shown here, this pattern continues outward in each direction.
The period of repetition is given in equation 4.

$$
\mathrm{s}=\mathrm{p} *(\mathrm{~d}+\mathrm{t}) / \mathrm{t}
$$

where:

$\mathrm{s}=$ lobe spacing

$\mathrm{p}=$ barrier period

$\mathrm{d}=$ normal distance from eyes to front screen plane

$\mathrm{t}=$ optical thickness between front and rear screens

The function of the two viewer control module is to set the barrier period such that this period is the minimum that produces no conflicts between actual eyes and virtual lobes. A quantity called conflict energy is defined by equation 5 for each eye, and total conflict energy for the system is defined by equation 6 .

$$
E_{i, j}=\left\{\begin{array}{l}
\left|e-d_{i, j}\right| ; \text { for } d_{i, j}<e \\
0 ; \quad \text { for } d_{i, j}>=e
\end{array}\right.
$$

where:

$E_{i, j}=$ conflict energy of eye $i$ with respect to eye $j$

$\mathrm{d}_{\mathrm{i}, \mathrm{j}}=$ distance from eye $\mathrm{i}$ nearest virtual lobe from eye $\mathrm{j}$

$\mathrm{e}=$ interocular distance

$$
\mathrm{E}_{\text {total }}=\sum_{\mathrm{i}} \sum_{\mathrm{j}} \mathrm{E}_{\mathrm{i}, \mathrm{j}}
$$

Equations 5, 6, and minimization algorithm can be summarized as follows. A conflict between an eye and a virtual lobe of another eye occurs when the distance between them is less than the interocular distance. When a conflict exists, the energy of that conflict is defined as the unsigned difference between the interocular distance and the conflicting distance. The total conflict energy is the sum of all possible conflict energies.

Because the total energy function contains many local minima, a linear search is required to compute the smallest barrier period where the total conflict energy becomes 0 . To optimize the search, parameters such as minimum, maximum, and step are tuned such that a small number of iterations $(<50)$ are performed for each frame.

\section{Synchronous / Asynchronous modes}

In order for the view distance, rapid steering, and 2-viewer modules to produce their intended results, Dynallax must rely on both synchronous and asynchronous communication and display modes. For example, when in rapid steering mode, communication needs to occur asynchronously so that the front and rear screens update at their own maximum possible rates. However, rapid steering is disabled with two viewers present and also momentarily when the barrier period is modified by the view distance module. The front and rear screens are synchronized under these circumstances so that barrier period changes occur as seamlessly as possible.

When Dynallax determines that a barrier period change is required, the slave processes rendezvous; the change is made using synchronous communication, and then the processes continue as they were. Whenever Dynallax switches to synchronous mode, an MPICH communication barrier is also employed as a secondary method of synchronizing the two slaves with each other. To summarize these operations, Figure 6 is a flow chart illustrating the two communication modes.

Communication in Dynallax follows a duplex handshaking protocol: slaves send ready messages to the master and the master 


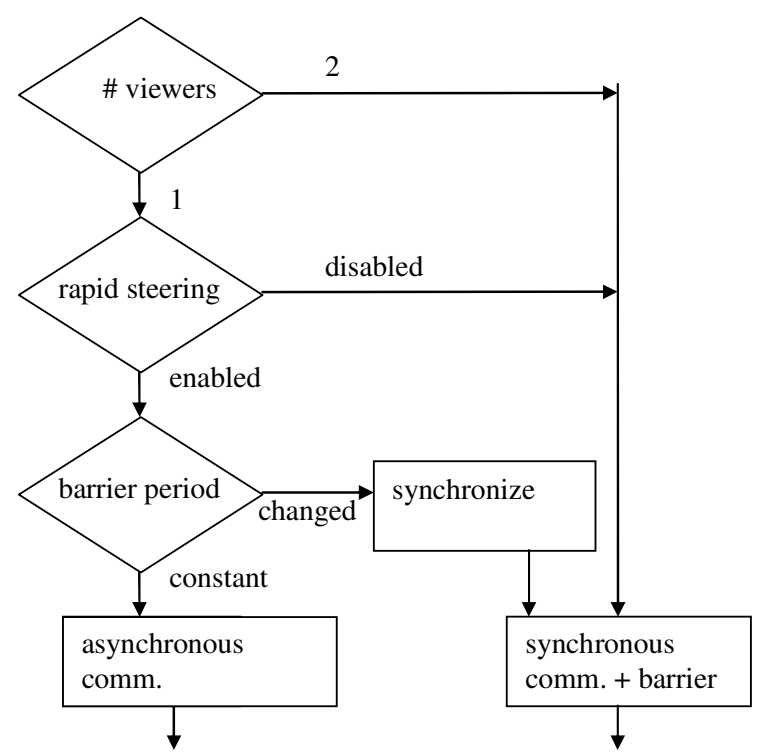

Figure 6: Inter-process communication in Dynallax is both synchronous and asynchronous.

responds by sending current data to the slaves. This flow control protocol permits slaves to run asynchronously when desired and to receive the most up-to-date information whenever they ask for it.

\section{Results}

\subsection{Barrier computation algorithm}

The Dynallax barrier is rendered and scenes are modulated using the sub-pixel shader algorithm of Section 3.3, with a barrier period that is continuously variable down to a theoretical minimum of one sub-pixel. In practice however, there are more restricted limits on the usable barrier range. These are a function of the physical front display screen, not the algorithm.

At one extreme, barrier periods of larger than about 12 pixels become visible to the viewer and divert attention from the scene to the barrier, while at the other, periods smaller than about 4 pixels cause noticeable ghosting because the steering of a colored sub-pixel of the rear screen is quantized by the front screen to the nearest pixel only. Since the front sub-pixels are colored rather than white, light from a rear sub-pixel of a given color can be transmitted only through certain locations of the front barrier; this restriction is elaborated upon in Section 5.

\subsection{View distance control}

In a parallax barrier strip system, the ghost level, or percentage of crosstalk, can be measured with a photometer by rendering one eye channel completely white and the other completely black. The difference in light levels reaching the eyes is then converted to percent ghost. In a static barrier system, the ghost level is at a minimum at the optimal view distance and increases when the viewer approaches the near and far view distance limits of the system. This is a particular disadvantage at the near viewing limit, because viewers often prefer to be close to the screen in order to see details.

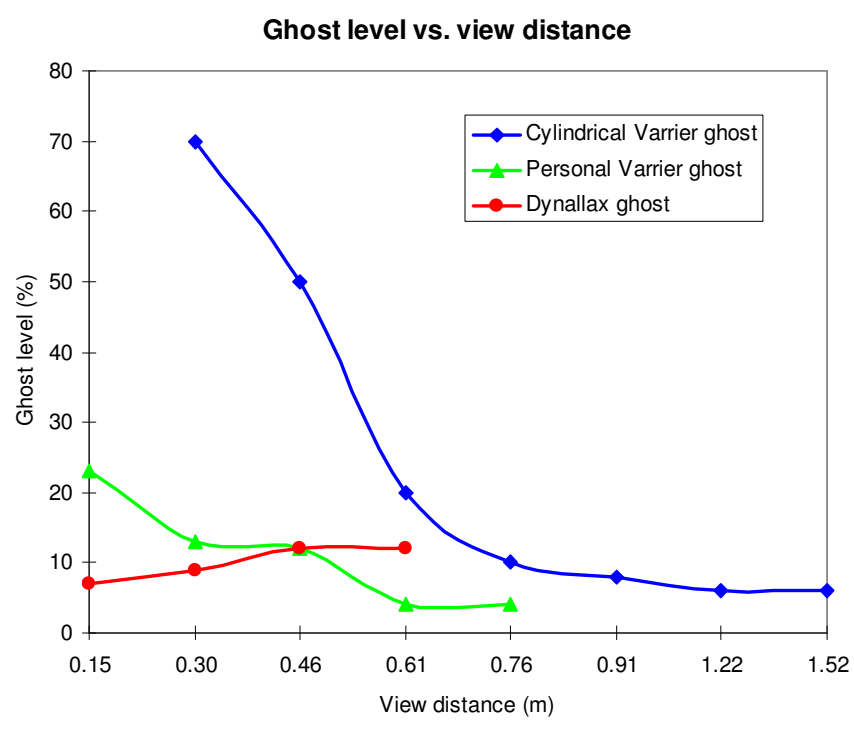

Figure 7: Ghost level vs. view distance is plotted for Dynallax and two Varrier implementations. Ghost level for a parallax barrier system can be measured with a photometer.

In Figure 7, the ghost level vs. view distance in the neighborhood of the near limit is plotted for two implementations of Varrier, the 35-panel Cylindrical Varrier [12] and the single panel Personal Varrier [8]. Because the Personal Varrier system is a seated desktop display, its barrier is tuned to a smaller minimum distance than the Cylindrical Varrier and hence its graph is shifted to the left, but the basic pattern is the same. On this is superimposed a graph of the ghost levels for Dynallax. All three systems have a comparable minimum ghost level of 5-7\%, but the Dynallax graph does not spike upward at near distances, as do the fixed barrier systems.

\subsection{Rapid view steering}

When rapid view steering is disabled, the front and rear screens operate in lockstep with each other at the same frame rate. This is the case for 2-viewer mode and also for various performance tests. On the other hand, with rapid view steering enabled, each screen is allowed to run as fast as possible and is updated with current data whenever the next frame is about to begin. The frame rate performance numbers in Table 1 bear this out for several model sizes.

Table 1: Frame rates for front and rear screens for various model sizes and rapid steering enabled / disabled

\begin{tabular}{|c|c|c|c|c|}
\hline$\underline{\text { Scene model }}$ & $\begin{array}{c}\text { single } \\
\text { polygon }\end{array}$ & Mars rover & head & skull \\
\hline$\underline{\text { \# vertices }}$ & 4 & $15 \mathrm{~K}$ & $130 \mathrm{~K}$ & $220 \mathrm{~K}$ \\
\hline$\frac{\text { front screen frame }}{\underline{\text { rate, synchronous }}}$ & $30 \mathrm{~Hz}$ & $30 \mathrm{~Hz}$ & $10 \mathrm{~Hz}$ & $3 \mathrm{~Hz}$ \\
\hline$\frac{\text { front screen frame }}{\text { rate, asynchronous }}$ & $50 \mathrm{~Hz}$ & $50 \mathrm{~Hz}$ & $50 \mathrm{~Hz}$ & $50 \mathrm{~Hz}$ \\
\hline$\frac{\text { rear screen frame }}{\text { rate }}$ & $30 \mathrm{~Hz}$ & $30 \mathrm{~Hz}$ & $10 \mathrm{~Hz}$ & $3 \mathrm{~Hz}$ \\
\hline
\end{tabular}


The purpose of rapid steering mode is to relax the sensitivity of parallax barrier AS VR to overall system latency. To show why this is the case, one may analyze the values in Table 1 by computing the maximum velocity of head movement permissible with and without rapid steering mode. Consider the skull model in the right column of Table 1. Furthermore, assume that head velocity is limited by a maximum movement of one half of the interocular distance $(32 \mathrm{~mm})$ during the time that a frame is displayed. This limit is reasonable because stereo is lost at this point; the eyes have moved completely into the guard bands before the scene is updated. Meanwhile, the time required to render a frame when the head is moving is given by the reciprocal of the frame rate in Table 1 plus tracker and communication latency, measured to be a constant $65 \mathrm{~ms}$ for a similar tracking system and cluster arrangement [12]. Given these values:

with rapid steering: $\quad \mathrm{v}=32 \mathrm{~mm} /(65+20 \mathrm{~ms})=.38 \mathrm{~m} / \mathrm{s}$ without rapid steering: $\mathrm{v}=32 \mathrm{~mm} /(65+333 \mathrm{~ms})=.08 \mathrm{~m} / \mathrm{s}$

The resulting speedup is a factor of greater than 4 times the permissible head velocity with rapid steering enabled.

\subsection{Two viewer mode}

Two viewers can each see their own stereo perspective of a scene by multiplexing four eye channels, directing one channel to each eye of each viewer, and setting the barrier period dynamically to minimize conflicts between the eyes and the virtual lobes. The barrier period for single viewer barrier at a view distance of approximately $.6 \mathrm{~m}$ is $1.2 \mathrm{~mm}$, so the minimum two-viewer barrier period would be twice as large, or $2.4 \mathrm{~mm}$. However, as Figure 8 demonstrates, in order to eliminate conflicts, barrier periods range from $2.9 \mathrm{~mm}$ to $5.2 \mathrm{~mm}$ for a variety of horizontal (x) displacements of the two viewers. Furthermore, Figure 8 reveals the irregular nature of the resulting barrier period function, reflecting the non-linearity of the underlying energy function in equations 5 and 6 .

The linear search time is not a bottleneck, but large barrier periods, when they occur, waste screen resolution. This is illustrated by the screen capture images in the lower portion of Figure 8. A test pattern is rendered with a different color for each of the four channels, and then the display is photographed from further back than the intended distance so that all four channels are visible simultaneously. When the barrier period is near $3 \mathrm{~mm}$ as in the lower left image, the channels are compactly juxtaposed, but large guard bands between some channels result when the period is larger, as in the lower right image.

Clearly, screen resolution is wasted when the barrier period and resulting guard bands expand, but this is required by the combination of positions of actual and virtual lobes in order to eliminate conflicts. Two improvements are possible to alleviate this condition. The baseline period for a single viewer can be reduced so that expansions due to two viewers begin with a smaller starting value. There are physical restrictions on the minimum possible single viewer period, and these are discussed in Section 5.

Another enhancement is that the controller algorithm may trade-off some small level of conflict energy with barrier period size. The tuning of the control algorithm remains as an ongoing research topic; presently the algorithm continues to increase the barrier period until the conflict energy drops to zero. However, near-zero conflict energies may result in smaller barrier periods with little increase in ghost level, and an alternate approach is to choose the smallest barrier period that results in some minimal conflict threshold.
Two-viewer barrier period

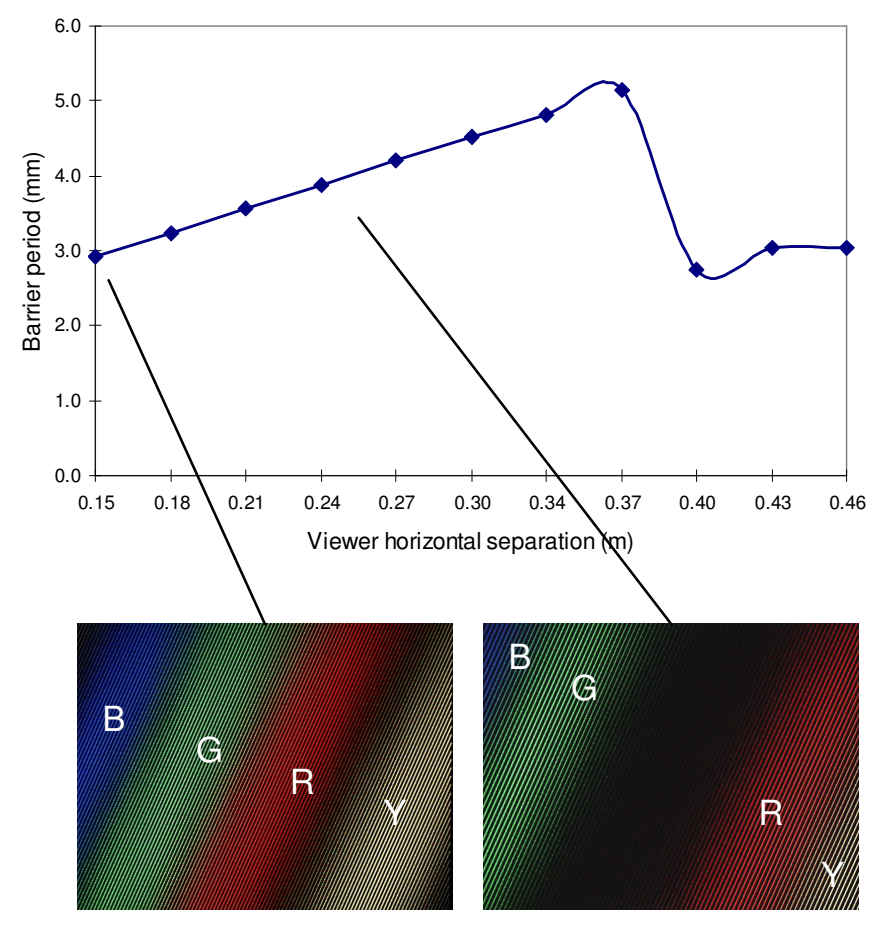

Figure 8: Two-viewer mode is tested with a variety of horizontal separations between the viewers. The conflict energy minimization algorithm produces the non-linear barrier period function shown in the graph above. In the screen capture images of test patterns below the graph, a different color channel is coded for each eye, $B$ = blue, $\mathrm{G}=$ green, $\mathrm{R}=$ red, $\mathrm{Y}=$ yellow. When the barrier period is near its minimum of $3 \mathrm{~mm}$, the channels are compactly spaced as

in the lower left image, but as the period grows, so does the

spacing between channels as in the lower right image. At a particular combination of viewer locations, channels are required to have certain spacing in order to avoid conflicts between physical and virtual lobes.

In order to observe quality in two viewer mode, an experimental apparatus is constructed consisting of two video cameras, separated by an interocular distance of $63 \mathrm{~mm}$ and fixed to a jig, thereby emulating the eyes of one viewer at a known 3D position in space. In Figure 9, a test pattern of vertical, horizontal, and angled bars is rendered for the various eye channels. In other words, each different angular orientation would correspond to a different eye's view of a VR scene in normal mode. The left column of Figure 9 is the left camera (eye) view and likewise for the right column and camera (eye). In $9 \mathrm{a}$ and $9 \mathrm{~b}$, only single viewer mode is enabled and horizontal bars are seen by the left eye and vertical bars by the right. The dim bars of the opposite orientation correspond to a ghost level of approximately 7\%. This is compared to figures $9 \mathrm{c}$ through $9 \mathrm{f}$, where two-viewer mode is enabled. $9 \mathrm{c}$ and $9 \mathrm{~d}$ correspond to the first viewer while $9 \mathrm{e}$ and $9 \mathrm{f}$ correspond to the second. The positions of first and second viewers are fixed in this test and the cameras are re-positioned between viewers. Comparing single and two-viewer modes, ghost level is observed to decrease slightly, however the barrier strips become more visible as their period is increased. More precise quantitative measurements of ghost level in two viewer mode under a variety of combinations of $3 \mathrm{D}$ positions of the two viewers are ongoing areas of study. 

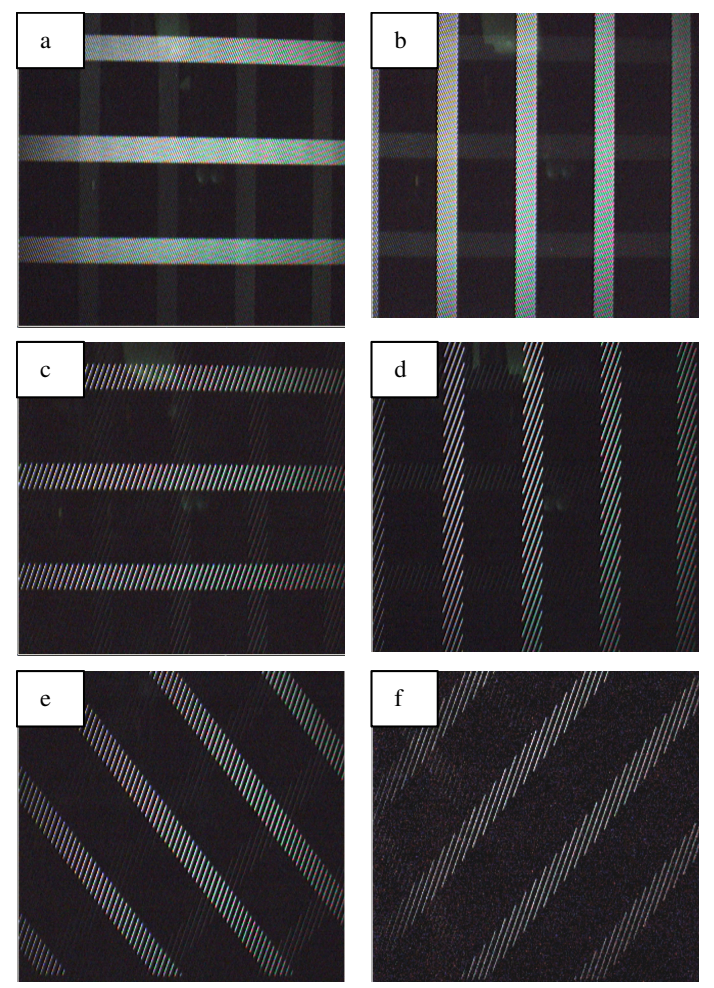

Figure 9: Left and right eye views under single and two-viewer modes are captured by cameras. $9 \mathrm{a}$ and $9 \mathrm{~b}$ : left and right eye of single viewer mode; $9 \mathrm{c}$ and 9d: left and right eye of first viewer under two-viewer mode; 9e and 9f: left and right eye of second viewer under two viewer mode. Different orientation white bars are rendered for the various channels, and the dim traces of opposite orientations are the ghost level of the system.

\subsection{Additional gains}

Earlier, it was mentioned that Dynallax is convertible between 2D and 3D modes. This is accomplished by rendering the front screen white, making it transparent, and rendering a single monoscopic view on the rear screen. This versatility permits the user to multiplex VR and non-VR tasks within the same display, even to the extent that the display space can be partitioned to permit both tasks to be visible simultaneously. Since no glasses are required to experience the 3D VR scene, the user can seamlessly perform both $2 \mathrm{D}$ and $3 \mathrm{D}$ tasks.

In static barrier strip implementations a registration process is required to align the physical barrier either with the underlying pixel structure, or in the case of Varrier, with the software's representation of the barrier computational model. Either way, it is a non-trivial process that is usually time-consuming, and the final quality of the system critically depends on the accuracy of this calibration process. This task is unnecessary in Dynallax as both front and rear screens are identical in structure, and the computational algorithms for both screens are highly correlated. However, if the front screen were constructed from a different size and type of LCD than the rear (as proposed in Section 5), then the calibration process would be required.

\section{CONCLUSIONS}

Through the use of a dynamic parallax barrier, Dynallax has been shown to improve the sensitivity to rapid head movement, permit two pairs of independent tracked perspective views, and extend view distance range. Additional benefits are the ability to disable the barrier and the elimination of barrier registration. Results have been quantified by measuring ghost levels, frame rates, and capturing screen images with a pair of cameras positioned at known locations and separated by an average interocular distance.

These advantages come at some cost though, and Dynallax still has some limitations that restrict its use to research at this time. The dual stacked LCD display is noticeably darker than a static barrier system, in fact both the brightness and contrast are approximately $50 \%$ that of a static system. Another limiting factor is the colored r,g,b sub-structure of the sub-pixels comprising the front screen. This limits the efficacy of the sub-pixel channel modulation algorithm below a barrier period size of one pixel.

If the sub-pixels were homogenously monochrome, eg. if the LC color filter could be removed, then the display could actually render barrier periods as low as one sub-pixel in channel width. Unfortunately, the color filter is an integral part of a modern LC panel. The result is that the sub-pixel resolution of the rear display is quantized to pixel resolution by the front display, since light of a given color can only pass through the same color front subpixel, as in Figure 10.

In the future, Dynallax will be tested with a monochrome medical-quality LCD panel serving as the front screen. It is expected that brightness and contrast will improve in this higher quality LC panel, and the net resolution of the system will increase due to the monochrome nature of the fine-pitch front pixels. The planned next step is to place a 2048x1538 monochrome 20-inch LC panel in front of a $1600 \times 120020$-inch color monitor.

One system parameter heretofore unmentioned is the spacing between the front and rear screens, called the optical thickness. Although the barrier period is dynamically variable, the optical thickness is not and is fixed by the physical separation when the stacked display is assembled. In future custom implementations, care must be taken when choosing the separation. A smaller screen separation results in a smaller barrier period by equation 2 .

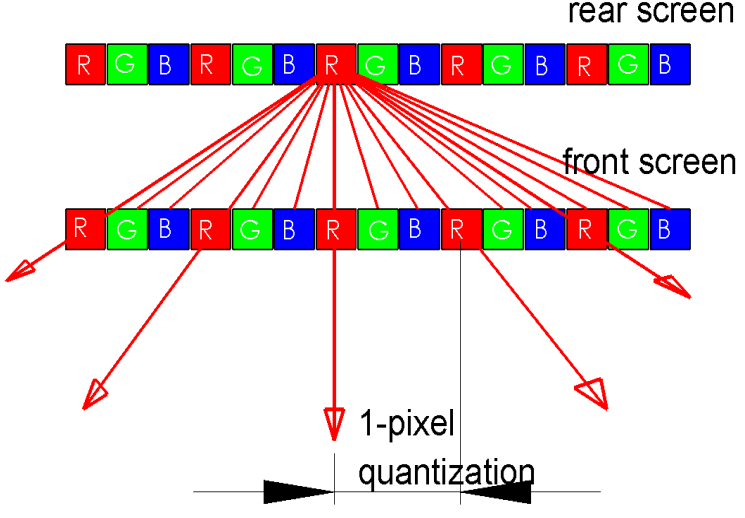

Figure 10: The colored sub-structure of the front display screen limits rear sub-pixel resolution to pixel resolution. 
This is desirable when near to the display or when two viewers are present, because at these times the period is automatically enlarged from this starting amount. However, when further from the screen, the barrier period computed by equation 2 must not drop below the physical limitations of the barrier display screen. This is true whether the screen is color or monochrome, although the value of the barrier limit is different for each. In general the barrier period cannot be less than the width of four or eight pixels or sub-pixels (for two or four views).

While the Dynallax prototype is driven by three separate machines, a more economical and compact future configuration will be a single machine with two separate graphics cards, not linked by SLI. The first card will drive the front screen and an optional console, while the second card will be dedicated to the rear screen. Although the prototype implementation of Dynallax consists of a single stacked display, the concept is scalable to tiled displays that will eventually incorporate the dynamic barrier concept in large wall-sized wide field of view displays.

\section{ACKNOWLEDGEMENTS}

The Electronic Visualization Laboratory (EVL) at the University of Illinois at Chicago specializes in the design and development of high-resolution visualization and virtual-reality display systems, collaboration software for use on multi-gigabit networks, and advanced networking infrastructure. These projects are made possible by major funding from the National Science Foundation (NSF), awards CNS-0115809, CNS-0224306, CNS-0420477, SCI-9980480，SCI-0229642，SCI-9730202，SCI-0123399，ANI 0129527 and EAR-0218918, as well as the NSF Information Technology Research (ITR) cooperative agreement (SCI0225642) to the University of California San Diego (UCSD) for "The OptIPuter" and the NSF Partnerships for Advanced Computational Infrastructure (PACI) cooperative agreement (SCI 9619019) to the National Computational Science Alliance. EVL also receives funding from the State of Illinois, General Motors Research, the Office of Naval Research on behalf of the Technology Research, Education, and Commercialization Center (TRECC), and Pacific Interface Inc. on behalf of NTT Optical Network Systems Laboratory in Japan. Varrier and CAVELib are trademarks of the Board of Trustees of the University of Illinois.

\section{REFERENCES}

[1] Javier Girado, Daniel Sandin, Thomas DeFanti, and Laura Wolf. Realtime camera-based face detection using a modified LAMSTAR neural network system. In Proceedings of SPIE-IS\&T Electronic Imaging, 20$24,2003$.

[2] InterSense. InterSense IS-900 Precision Motion Tracker. http://www.isense.com/products/prec/is900/, 2006.

[3] Robert Kooima. Varrier Combiner. http://www.evl.uic.edu/rlk/varrier_combiner/varrier_combiner.html, 2006. [4] Robert Kooima et al. Varrier Combiner, paper submitted for publication to IEEE VR, 2007. (under review)

[5] Lenny Lipton and Mark Feldman. A New Autostereoscopic Display Technology: The SynthaGram. In Proceedings of SPIE Photonics West 2002: Electronic Imaging, San Jose, California., 2002.

[6] MPICH2. http://www-unix.mcs.anl.gov/mpi/mpich/, 2006.

[7] Takanori Okoshi. Three Dimensional Imaging Techniques. Academic Press, N.Y., 1976.

[8] Tom Peterka, Daniel Sandin, Jinghua Ge, Javier Girado, Robert Kooima, Jason Leigh, Andrew Johnson, Marcus Thiebaux, and Thomas DeFanti. Personal Varrier: Autostereoscopic Virtual Reality for
Distributed Scientific Visualization. In Future Generation Computing Systems, vol. 22, no. 8, p. 976-983, 2006.

[9] PureDepth. http://www.puredepth.com, 2006.

[10] Randi Rost. OpenGL Shading Language. Redwood City, CA, Addison-Wesley Longman, 2004.

[11] Daniel Sandin, Todd Margolis, Greg Dawe, Jason Leigh, Thomas DeFanti. The Varrier Autostereographic Display, In Proceedings of SPIE, vol. 4297, San Jose, California, 2001.

[12] Daniel Sandin, Todd Margolis, Jinghua Ge, Javier Girado, Tom Peterka, and Thomas DeFanti. The Varrier ${ }^{\mathrm{TM}}$ autostereoscopic virtual reality display, In ACM Transactions on Graphics, Proceedings of ACM SIGGRAPH, 24, no. 3, 2005, p. 894-903, 2005.

[13] Alexander Schmidt and Armin Grasnick. Multi-viewpoint Autostereoscopic Displays from 4D-Vision. In Proceedings of SPIE Photonics West 2002: Electronic Imaging, San Jose, California, 2002. [14] David Montgomery, Graham Woodgate, Adrian Jacobs, Jonathan Harrold, and David Ezra. Analysis of a flat panel display system convertible between 2D and autostereoscopic 3D modes. In Proceedings of SPIE, 4297, p. 148-159, 2001.

[15] Ken Mashitani, Goro Hamagishi, Masahiro Higashino, Takahisa Ando, and Satoshi Takemoto. Step barrier system multi-view glass-less 3D display. In Proceedings of SPIE-IS\&T Electronic Imaging, 5291, p. 265-272, 2004.

[16] Richard Moseley, Graham Woodgate, and David Ezra. Observer tracking directional display. United States Patent 6377295, 2002.

[17] Neil Dodgson, John Moore, Stewart Lang, Graham Martin, and Peter Canepa. A 50" time-multiplexed autostereoscopic display. In Proceedings of SPIE Electronic Imaging, 3957, 2000.

[18] Ken Perlin, Salvatore Paxia, and Joel Kollin. An Autostereoscopic Display. In Proceedings of ACM SIGGRAPH 2000, Computer Graphics Proceedings, Annual Conference Series, p. 319-326, 2000. 